\title{
La SFP et les « jeunes » physiciens et physiciennes
}

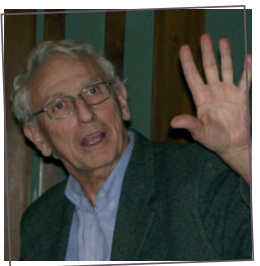

Sur les presque 3000 membres de la SFP, 20\% à peine sont des " jeunes " : étudiants en master ou en école d'ingénieurs, doctorants et post-doctorants, jeunes maitres de conférences et chargés de recherche ou ingénieurs de moins de 35 ans. Ce chiffre n'est pas satisfaisant et représente moins de $10 \%$ de cette communauté de jeunes physiciens. Nos grandes sociétés sœurs : la Deutsche Physikalische Gesellschaft (DPG), en Allemagne, et I'Institute of Physics (IOP), au Royaume-Uni, réalisent de bien meilleures performances. La moitié des membres de la DPG (50 ooo membres) ont moins de 35 ans ; il en est de même pour le quart des 40000 membres de l'IOP, alors que les communautés de physiciens et d'ingénieurs sont de taille comparable à celle de la France. Parmi les multiples activités de la DPG, celles destinées aux jeunes chercheurs ont beaucoup de succès : elles proposent des rubriques « recherche d'emploi » dans le magazine et sur le site Internet de la DPG, et aussi des contacts avec les entreprises. Côté IOP, plusieurs actions spécifiques sont proposées aux étudiants, telles qu'une information complète sur le système de bourses et une aide à la rédaction de CV.

La SFP a pris conscience de la nécessité de recruter des "jeunes", en particulier au séminaire d'Orléans en décembre 2005. Aux Journées de la Matière Condensée de Toulouse en août 2006, une table ronde sur l'emploi des docteurs en physique a été organisée en présence d'industriels. Il en sera de même au Congrès Général de la SFP à Grenoble en juillet
2007. Notre collègue Johannes Orphal coordonne plusieurs initiatives en vue de mieux répondre à l'attente des "jeunes " : citons la création de rubriques " offres et demandes d'emploi » sur le site rénové de la SFP et un espace réservé aux jeunes physiciens dans "Reflets de la Physique ", qui démarre avec des interviews de jeunes chercheurs et des articles d'industriels sur le problème du recrutement. Un rapport détaillé sur la situation de l'emploi des docteurs en physique est accessible sur le blog de H. Bartholin : http://physicistemployment.blogspot.com.

Un réseau de correspondants "jeunes " dans les sections régionales est en cours de constitution. Les nouveaux adhérents pourront contribuer à travers la SFP à des débats sur les problèmes de société concernant la physique (énergie, environnement,...), sur la politique de la recherche nationale et européenne, sur les problèmes de l'enseignement de la physique, sur les problèmes d'éthique.

Une campagne d'adhésion à prix réduit est mise en place : elle est décrite à la fin de cet éditorial. Puisque cet article sera plutôt lu par les membres " non jeunes " (désolé pour ce qualificatif, cher lecteur !...), nous vous incitons fortement à faire adhérer vos jeunes collaborateurs dans vos laboratoires. C'est vital pour la SFP : si notre société est assez puissante, elle pourra alors intervenir plus efficacement auprès des décideurs en faveur des moyens de la recherche et des carrières.

Roger Maynard 